\title{
Acoustic tomography in the atmospheric surface layer
}

\author{
A. Ziemann, K. Arnold, A. Raabe \\ Institute of Meteorology, University of Leipzig, Stephanstr. 3, D-04103 Leipzig, Germany \\ E-mail: ziemann@rz.uni-leipzig.de
}

Received: 20 January 1998 / Revised: 27 May 1998 / Accepted: 9 June 1998

\begin{abstract}
Acoustic tomography is presented as a technique for remote monitoring of meteorological quantities. This method and a special algorithm of analysis can directly produce area-averaged values of meteorological parameters. As a result consistent data will be obtained for validation of numerical atmospheric micro-scale models. Such a measuring system can complement conventional point measurements over different surfaces. The procedure of acoustic tomography uses the horizontal propagation of sound waves in the atmospheric surface layer. Therefore, to provide a general overview of sound propagation under various atmospheric conditions a two-dimensional ray-tracing model according to a modified version of Snell's law is used. The state of the crossed atmosphere can be estimated from measurements of acoustic travel time between sources and receivers at different points. Derivation of area-averaged values of the sound speed and furthermore of air temperature results from the inversion of travel time values for all acoustic paths. Thereby, the applied straight ray two-dimensional tomographic model using SIRT (simultaneous iterative reconstruction technique) is characterised as a method with small computational requirements, satisfactory convergence and stability properties as well as simple handling, especially, during online evaluation.
\end{abstract}

Key words. Meteorology and atmospheric dynamics (turbulence; instruments and techniques).

\section{Introduction}

The development of micro-scale atmospheric and LES (large eddy simulation) models (e.g., Deardorff, 1974; Moeng, 1984; Shaw and Schumann, 1992), which are

Correspondence to: A. Ziemann applied for different questions in meteorology, forces their validation by means of accurate experimental data and derivation of area-averaged meteorological quantities. Spatially averaged data, required for consistent model validation, were conventionally provided by point measurements and a subsequent interpolation technique. A relatively new way to obtain such values directly is the application of tomographic methods to the atmospheric surface layer (see Spiesberger and Fristrup, 1990; Wilson and Thomson, 1994).

In our study we use a kind of acoustic travel time tomography, where the sound speed can be determined by measuring the travel time of a signal along a defined propagation path. Applying a suitable procedure, measurements of the sound speed can be used to reconstruct the spatially and temporally variable temperature and wind fields. Tomography is the cross-section construction of a test object using the reaction of the considered medium on an external probing energy (see Lo and Inderwiesen, 1994). The higher the number of measuring points, the higher the spatial resolution for the estimated meteorological parameters. As an inverse technique tomography has been used routinely for instance in medicine, biology, geophysics and oceanography for many years (see, e.g., Munk et al., 1995). There are various advantages of tomographic measurements compared with conventional methods, for instance the effect as a spatial filter for turbulence, the remote monitoring aspect (because the test medium is not influenced by devices) and the higher number of data per sensor in comparison to traditional point measurements (Wilson and Thomson, 1994).

Since the end of the 1960s matrix inversion methods have been applied in geophysics. Ten years ago algebraic reconstruction techniques, for instance SIRT (simultaneous iterative reconstruction technique), began to play an important role, especially due to their small computational requirements.

One condition for tomography is a medium with constant properties during measuring time. Therefore, by an application of tomographic methods on the 
atmosphere, measurements of acoustic travel time have to be executed in the same time from all directions on relatively short distances between the experimental devices. Measurements of travel time must be carried out on different ray paths between accurately positioned sources and receivers. Then just the inversion of a single set of measurements obtained from different points of view results in a statement about the spatial distribution of quantity values investigated.

Wilson and Thomson (1994) demonstrated the applicability of acoustic tomography to the atmospheric boundary layer. The significant difference compared to the work presented here lies in the numerical algorithm and in the kind of the output. We apply the tomographic technique SIRT to provide from the measured line integrals, the travel time data, not only differences from a mean value, but also area-averaged absolute values for the sound speed and further for the air temperature and wind velocity. Our measuring system, which can be used for a wide range of environmental and equipment situations, is introduced as a method to complete meteorological point measurements, especially, during experimental campaigns.

The following section describes some fundamentals of the sound propagation in the atmosphere. In sect. 3, a general view of tomographic methods, and especially of the SIRT method used in our study, is given. The next section deals with the implementation of the presented principles of geometric acoustics and tomography in numerical models, namely a two-dimensional ray-tracing model for a stratified atmosphere and a tomographic model according to a horizontal-slice scheme. In sect. 5, the experimental equipment and the processing of acoustic signals, as well as the demanded accuracy of the travel time data, are described. In sect. 6, the results of our first experimental campaign in autumn 1996 and an outlook towards necessary improvements are supplied.

\section{Theoretical bases of sound propagation in the atmosphere}

In addition to the well-known reduction of the sound level with increasing distance from the sound source, the influence of inhomogeneities within the atmosphere, damping due to the structure of the ground, and air absorption, all play a role during the sound propagation through the atmosphere. If dry air is considered as ideal gas, Laplace's equation of sound speed can be used under adiabatic conditions:

$c=\sqrt{\gamma_{a} R_{a} T}$,

where $\gamma_{a}(=1.4)$ is the specific-heat ratio, $R_{a}$ is the dry adiabatic gaseous constant, and $T$ is the air temperature.

Effects of air humidity (e.g., Spiesberger and Fristrup, 1990) or a modified air composition on sound velocity are neglected because typical changes are less than $0.05 \%$. In addition to spatial (Cartesian coordinates $x, y, z$ ) and temporal (time $t$ ) fluctuations in the air temperature, the wind field modifies the sound velocity and leads to an effective sound speed vector using the approximation of wave fronts (see Pierce, 1989) from geometrical acoustics with $\mathbf{n}$ as unit vector normal to the wave front:

$c_{\text {eff }}(T, v)=c(T(t, x, y, z)) n(t, x, y, z)+v(t, x, y, z)$

Thereby, the conditions for sound propagation are changed and so a deviation from straight line path between sound sources and receivers can be observed.

The sound propagation can be described with sound rays if the principles of geometric acoustics, i.e., small acoustic refraction index gradient compared to the wave number of the sound wave (e.g., Klug, 1991), are applicable. This case often occurs under outdoor conditions, except in the immediate vicinity of the ground. At frequencies above $100 \mathrm{~Hz}$, sound propagation can usually be simulated with ray theory. Therefore, the ray equation is often referred to as the high-frequency solution to the wave equation (see Pierce, 1989). Although the use of the sound ray theory, which is based on the principles of Fermat and Huygens, is associated with limitations, it offers, in comparison to wave models, the following advantages: (1) an easy visualisation of sound propagation inside the atmosphere; and (2) a simple consideration of inhomogeneities inside the medium by application of the refraction law.

When there is a gradient in the sound speed or wind velocity, sound rays are refracted. In the atmosphere this refraction is due mainly to variations in air temperature and wind vector depending on altitude in stratified fluids. From Snell's law for unmoved media a general form can be derived for stratified media with wind velocity in direction of the sound speed (see, e.g., Birkhoff, 1988; Kornhauser, 1953). The derivation of the vector Eikonal equation, for a moving and inhomogeneous medium, is shown, for instance, by Ugincius (1965). He demonstrates a simple solution in the case of a stratified fluid, such as described already, leading to an equation for the ray path. This result agrees with a derivation given by Fasold et al. (1984) which is used in this study. Assuming the same frequency $f$ in the two media 1 and 2 it follows:

$\sin \alpha_{2}=\frac{k_{1} \sin \alpha_{1}}{k_{2}+\left(k_{2}\left|\mathbf{M a} \mathbf{a}_{1}\right| \cos \varphi-k_{1}\left|\mathbf{M a} \mathbf{a}_{2}\right| \cos \varphi\right) \sin \alpha_{1}}$

with $\left|\mathbf{M a}_{\mathbf{1}}\right|=\frac{\left|v_{1}\right|}{c_{2}},\left|\mathbf{M a}_{2}\right|=\frac{\left|v_{2}\right|}{c_{1}}, k_{1}=2 \cdot \pi \cdot \frac{f}{c_{1}}, k_{2}=2 \cdot \pi \cdot \frac{f}{c_{2}}$ Thereby, $\alpha_{1}$ is the incident and $\alpha_{2}$ is the refracting angle, $\varphi$ is the angle between wind direction and undisturbed (without wind influence) sound velocity vector, $\mathbf{M a}_{\mathbf{1}}$ and $\mathbf{M a}_{2}$ are the Mach numbers, $k_{1}$ and $k_{2}$ are the wave numbers. Note that the angles $\alpha_{1}$ and $\alpha_{2}$ are related to the wave normal in the medium 1 and 2 , respectively.

Other atmospheric influences, like changes of the sound pressure level caused by scattering of the sound waves on temperature inhomogeneities and turbulence elements, as well as the damping in consideration of air absorption, amount to about $0.01 \mathrm{~dB}$ for the used frequency range between $500 \ldots 1000 \mathrm{~Hz}$, and sourcereceiver distances between $75 \ldots 230 \mathrm{~m}$, according to 
numerous authors (Tatarskii, 1961; Kneser, 1961; Aubry et al., 1974; Daigle et al., 1978; Bass, 1981). Therefore, these processes can be neglected for our travel time measurements with a $110 \mathrm{~dB}$-sounder and ray paths up to $230 \mathrm{~m}$.

\section{Travel time tomography}

The theoretical basis of tomography lies in the Radon transformation (Radon, 1917; Chapman, 1987) related to the projection of a two-dimensional scalar field and the transformation from the measured value distribution to the projection field. According to Radon's law it is possible to reconstruct a spatial distribution of parameters by inversion of line integral values, e.g., measured travel times. Hence, a continuously two-dimensional function can be derived from an infinite number of its continuous one-dimensional projections. The task is to determine the original distribution of parameter values from the measured projections. Thereby, the projectionslice scheme will be applied (e.g., Rüter and Gelbke, 1986). The general principle of tomography (from Greek 'tomos' meaning slice or section), as a certain image reconstruction technique and solving method for inversion problems, is to create a model of physical parameters in such a way that the projected data agree with the measured data.

Review of first applications of acoustic tomography to the atmosphere is given by Wilson and Thomson (1994). The historical development, especially, in seismic applications of tomography, is presented by Humphreys and Clayton (1988). Recently tomographic methods of sound propagation in the atmosphere were applied by Spiesberger and Fristrup (1990), who described a method for passive localisation of calling animals, and by Wilson and Thomson (1994), who primarily include the characterisation of the atmosphere. The main differences of these works to our study exist in the experimental design, the signal processing, and the interpretation algorithm including the tomographic method. Both our study and the one from Wilson and Thomson (1994) use horizontal-slice schemes, and therefore are two-dimensional tomography. The advantages of our procedure are the small computational time and memory requirements, as only one ray is in the memory at one time. Therefore, it is also possible to use our procedure for online interpretation of measurement results during experimental studies. According to Worthington (1984), the procedures to solve tomographic problems can be divided into three main groups. Direct analytical solutions based on Fourier or inverse Radon transformation are usually employed for biological and medical applications with a homogeneous, high density ray coverage. Alternative methods were developed, e.g., in geophysics and oceanography, where often such ideal ray and angle coverage does not occur.

One possible algorithm to solve the linear equation system with the desired parameter values as unknown variables is the matrix inversion method. Thereby, the generalised matrix inversion is the most important procedure if there are more unknowns than linearly independent equations (see Aki and Richards, 1980; Backus and Gilbert, 1968). Because of the space- and time-consuming handling of large matrixes, which have to be inverted, the practical applicability of this method is limited (Krajewski et al., 1989). Consequently, methods referred to as row action (Trampert and Leveque, 1990) or ART (algebraic reconstruction technique), in which the approximate solution is updated by successively processing the equations, can be more attractive.

A large number of iterative reconstruction techniques (e.g., Peterson et al., 1985) can successfully be used for different measurement geometries with irregular sampling or limited projection angles with the additional advantage of simple handling and small computational requirements. The most prominent methods are ART and SIRT according to Gordon et al. (1970) and Gilbert (1972), respectively. A comprehensive review of the mathematical background of ART-like methods is given by van der Sluis and van der Vorst (1987).

An early example of such techniques is Kaczmarz's method (1937). Improvements according to Dines and Lytle (1979) lead to a comparable better convergence behaviour. Such procedures (see van der Sluis and van der Vorst, 1987), in which the approximate solution is updated only after all equations have been processed, are called SIRT (Trampert and Leveque, 1990).

Other techniques, such as Wiener filtering (also called objective analysis or Kriging), parameter estimation schemes, and Kalman filters, are recommended (see Spiesberger and Fristrup, 1990; Wilson and Thomson, 1994) to obtain a solution for the deviation from a starting model. That means, relations between fluctuations in the measured data and fluctuations in the model of the searched parameter are obtained. Thereby, additional information, e.g. about the covariance lengths, have to be available. In our study we want to measure absolute values, therefore, a method from the large ART-family, namely SIRT, is preferred.

Principally, an observed data set consists of line integrals along a specified path of the parameter to be imaged, e.g., acoustic travel time, which contains information about the physical quantities of the medium. Inhomogeneities of air temperature or in the wind field inside the tomographic array influence each measured travel time in an other way. Each measurement is ambiguous, and only the inversion of every travel time measurement produces an image of the medium features radiated through with sound rays.

For a two-dimensional geometry the line integral for the travel time $\tau$ of a signal between a fixed acoustic source and receiver can be written

$\tau=\int_{\text {ray }} \frac{\mathrm{d} l}{\left|\mathbf{c}_{\text {eff }}\right|(t, x, y)}=\int_{\text {ray }} s(t, x, y) \mathrm{d} l$,

where $\mathrm{d} l$ is the element of arc length along the propagation path and $s$ symbolises the slowness, i.e., the reciprocal effective sound velocity. Fundamental difficulties thereby are that the ray path itself depends on the unknown slowness distribution and that the line 
integral becomes non-linear in slowness. Usually, a linearisation is applied to get some initial slowness model and to solve this problem. Thereby, straight lines connecting the sound source and the receiver are used to approximate the true ray path. This approximation is valid according to Fermat's principle. As a consequence of this principle perturbations in ray paths are second order with respect to perturbations in slowness (see Bording et al., 1987; Bregman et al., 1989a).

In practice only a limited number of measurements is available. Therefore, the desired two-dimensional function can only be reconstructed as a discrete parameter distribution, i.e., constant parameter values inside the grid cells. Various discretisations are possible. We use a tomographic array covered with square cells of constant size. The linearised set of equations in discrete form follows that of Rüter and Gelbke (1986)

$\tau_{i}=\sum_{j=1}^{J} s_{j} l_{i j} \quad$ with $s_{j}=\frac{1}{c_{j}}$,

where $\tau_{i}$ is the travel time of the $i^{\text {th }}$ sound ray, $s_{j}$ and $c_{j}$ are the slowness and the effective sound speed in grid cell $j$, respectively, and $l_{i j}$ is the length of the ray piece of the ray $i$ in the $j^{\text {th }}$ cell, or with the column vector $\mathbf{T}$, the $(\mathbf{I} \times \mathbf{J})$ matrix $\mathbf{L}$ of ray segments and the row vector $\mathbf{S}$

$\mathbf{T}=\mathbf{S} \cdot \mathbf{L}$.

Since matrix $\mathbf{L}$ is typically large, formal matrix inversion consumes a relatively large amount of computational time and memory. A common technique for solving such systems are iterative reconstruction techniques, for instance SIRT used in our study. An image of diverse properties of the domain, which was radiated through from several angles of view, can be deduced by the following inversion technique. Thereby, the measuring field is divided into small area elements. Figure 1 shows the partitions $\left(20 \times 20 \mathrm{~m}^{2}\right)$ of the tomographic array $\left(100 \times 200 \mathrm{~m}^{2}\right)$ of the field experiment ACTO'96.

The dimension of the grid cells was chosen depending on the number of sound rays and the desired resolution. For each grid cell constant values of the slowness and the sound speed, respectively, were assumed. From the mean travel time $\tau_{i}$ information about the searched travel time and slowness in each grid cell $j$ can be extracted using the known ray path $l_{i j}$. The aim of the following procedure is the reconstruction of the slowness $s_{j}$. In our study we applied a kind of straight ray tomography, the simplest ray-tracing between sources and receivers. The error made by this approximation is investigated with a ray-tracing model which requires highly resoluted vertical profiles of meteorological quantities. This model is described in sects. 2 and 4.

An example for a straight ray imaging technique is the back projection (BP) algorithm (see Bregman et al., 1989a; Humphreys and Clayton, 1988) which yields a model with a smoothed and often blurred slowness field. Thereby, artefacts can occur due to a discrete data sampling. To improve this initial model various iterative reconstruction techniques can be used, for instance

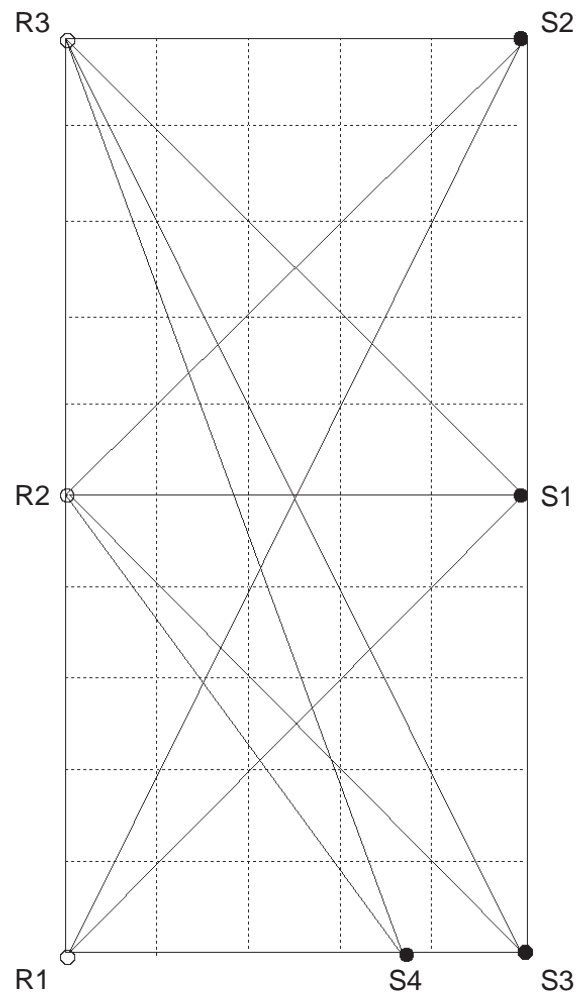

Fig. 1. Layout of the tomographic array $\left(100 \times 200 \mathrm{~m}^{2}\right)$ showing the grid cells $\left(20 \times 20 \mathrm{~m}^{2}\right)$. Sources are labeled $\mathrm{S} 1$ to $\mathrm{S} 4$, and receivers are labeled R1 to R3

SIRT (e.g., Herman, 1980) as the most convenient method because of its speed and stability.

For every algebraic reconstruction technique a forward model has to be proposed in order to get the searched distribution by an iterative comparison process between calculated and experimental data. Starting with an initial guess of the slowness values, $s_{i}^{0}$, SIRT iterates on the difference between the experimentally obtained travel time $\tau_{i}^{\text {measure }}$ and the last model prediction $\tau_{i}$. After back projection of this difference, and adding the resulting correction to the present model, an updated version of the simulated travel time follows. Thereby corrections are carried out to meet the condition:

$\tau_{i}^{\text {measure }}=\sum_{j=1}^{J}\left(s_{j}+\bar{k}_{j}\right) l_{i j}$

From the distribution of the differences

$\Delta \tau_{i}=\tau_{i}^{\text {measure }}-\tau_{i}$

among all grid cells an averaged value for correction $\bar{k}_{j}$, corresponding to the minimum energy criterion, will be obtained after multiplication with the ray length $l_{i j}$ (according to Dines and Lytle, 1979; McMechan, 1983) as well as after the treatment of all rays which touch the respective cell $N_{j}$ times:

$k_{j}=\Delta \tau_{i} \frac{l_{i j}}{\sum_{j=1}^{J} l_{i j}^{2}} \Rightarrow \bar{k}_{j}=\frac{1}{N_{j}} \sum_{j=1}^{J} k_{j}$ 
This process is continued until attainment of a convergence criterion. Thereby, SIRT converges to the leastsquares solution (see Ivansson, 1983).

With a changed denominator in the form $\left(\sum_{j=1}^{J} l_{i j}\right)^{2}$ in Eq. (9) the convergence is slowed down by a factor of about 10 (see Gelbke, 1988), in comparison to the applied form of Eq. (9).

The resulting set of area averaged values of slowness and sound velocity is called a tomogram.

According to Santamarina and Reed (1994) the fidelity and quality of inversions are affected by the nature of the analysed phenomenon, the quality of measured data, and the applied inversion procedure. Errors in the data set can lead to a tomogram with an artificial chess board pattern during the iteration cycle, particularly, without an averaging process of the corrections $k_{j}$. A minimum difference between the measured and simulated data is only a necessary, but not a sufficient, condition for the convergence to the correct model. For strongly erroneous data only a solution with a maximum entropy can be found. The larger the distance between the source and receiver, the greater is the measuring effect and the influence of the measurement to the tomographic solution. Therefore, the accuracy of the signal and data analyses as well as the positions of the sources and receivers are very important for successful tomography.

One problem is also the limited angular coverage of the tomographic array which causes a poor resolution of structures oriented perpendicular to ray paths (see McMechan, 1983; Gelbke, 1988). Incomplete angular coverage can be balanced by imposed constraints, as some authors have done in different ways (e.g., Carrion, 1991). In medical applications, however, it is possible to produce isotropic and homogeneous ray coverage. For an application in the atmosphere, usually both of these properties do not hold. The non-ideal ray distribution can lead to streaks that radiate from anomalous blocks along the direction taken by the rays traversing these blocks. By reducing the weight given rays aligned in common orientations this effect can be reduced (see Humphreys and Clayton, 1988). Only anomalies bordered and crossed by ray paths can accurately be reconstructed, otherwise a smearing of the inhomogeneity can occur. Furthermore, possible artefacts in the solution can be caused by badly sampled cells and nonideal ray geometry, particularly, for longer rays (Trampert and Leveque, 1990). Damping, slowness constraints, or the use of other a priori information effectively removes possible problems due to the underdetermination of the system of equations (see Bregman et al., 1989b; Krajewski et al., 1989). Therefore, we have also to deal with such questions in the future to improve our tomographic results.

\section{Numerical modelling of the sound propagation and travel time tomography}

Under special conditions, given in sect. 2, sound propagation through the atmosphere can be described with a ray-tracing model. In the present study a twodimensional $(x-z)$ ray-tracing model for a homogeneous atmosphere in the horizontal direction is applied to determine the principal characteristics of sound propagation in the atmosphere and the difference between a straight and curved ray according to actual conditions. It is assumed in our model that the sound propagation follows the $x$-direction (see Raabe et al., 1996) in a Cartesian co-ordinate system. The atmosphere is thereby divided vertically into layers having thickness $\Delta \mathrm{z}$. Equation (3) and the relations

$$
\begin{array}{r}
\Delta s=\sqrt{\Delta x^{2}+\Delta z^{2}} \\
\Delta x=\Delta z \cdot \tan \alpha_{2}=\Delta z \frac{\sin \alpha_{2}}{\sqrt{1-\sin ^{2} \alpha_{2}}}
\end{array}
$$

are used for the calculation of the ray path $\Delta s$ inside the vertical and horizontal range $\Delta z$ and $\Delta x$, respectively. Vertical temperature and wind profiles from a numerical atmospheric boundary layer model (see, e.g., Mix et al., 1994; Ziemann, 1998) with high resolution of a few centimetres in the lowest $20 \mathrm{~m}$, or profiles from experiments (i.e., from a meteorological mast) are used as input values for the ray-tracing model.

Up to now area-averaged values can be considered by a straight ray two-dimensional $(x-y)$ tomographic model. Therefore, the influence of the curved ray path in the vertical direction has to be estimated with the raytracing model. The discrepancies between the actual calculated ray path, which is influenced by the angle of emission, and the exactly straight ray path between the source and the receiver amount to about $0.20 \mathrm{~m}$ for a source-receiver-distance of about $200 \mathrm{~m}$ (see Fig. 2). This value agrees well with the results obtained by Spiesberger and Fristrup (1990) for a linear profile of the sound speed under typical daytime conditions.

The basic set of equations used in the tomographic model was already explained in the previous section. One disadvantage of SIRT is the unavailable information about the resolution matrix, $\mathbf{R}=\mathbf{L}^{\mathrm{T}}\left(\mathbf{L} \mathbf{L}^{\mathbf{T}}\right)^{-1} \mathbf{L}$

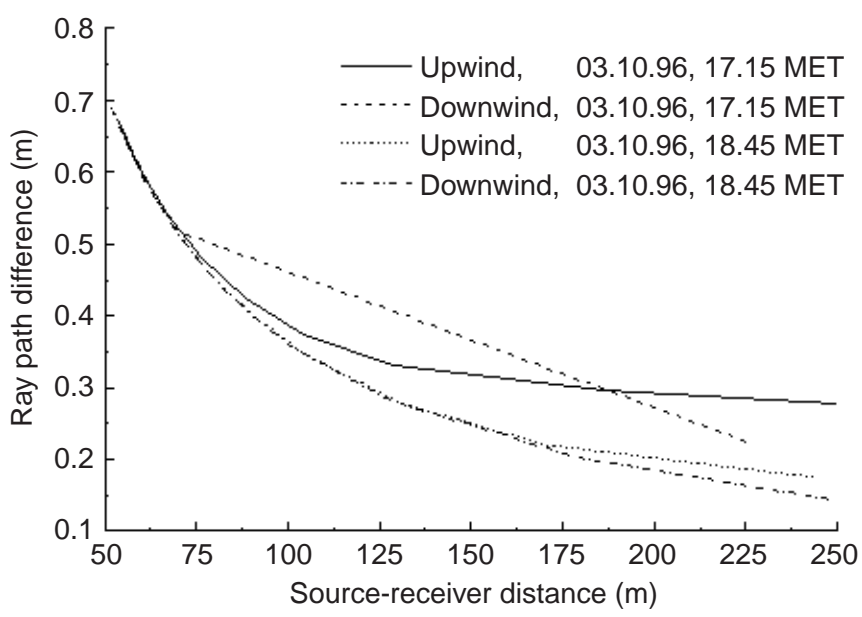

Fig. 2. Differences between the actual calculated ray path from the ray-tracing model and the exactly straight ray path between the source and the receiver as a function of the source-receiver distance 
according to Backus and Gilbert (1968). A quantitative measure of the similarity of the reconstructed image and the model is defined by the Euclid distance $(E D)$ with the actual model slowness, $s_{j}$ and the current estimate, $s_{\dot{j}}^{n}$, from the $n^{\text {th }}$ iteration cycle (see Krajewski et al., 1989) $E D=\frac{1}{J} \sum_{j=1}^{J}\left(s_{j}-s_{j}^{n}\right)^{2}$. It is obvious that the inversion result becomes better for smaller than for larger $E D$.

To avoid divergence of the solution and formation of artefacts due to erroneous data, the number of iterations should be limited corresponding to the optimal reconstruction result. Therefore, the sum of squared residuals (SSR) from Eq. (8) is a quantity describing the progress of the solution of the equation system. The optimal image will be achieved if the current decrease of SSRvalues, averaged over five iteration cycles, diminishes to about $1 \%$ of the decrease during the first five steps (see Krajewski et al., 1989). In our study this value is reached not later than after 100 iterations running in about one minute on a PC (100 MHz Pentium processor).

The size of the grid cells (see Fig. 1) has to be chosen so that on the one hand area elements are crossed by a relatively large number of rays, and on the other hand the desired resolution for searched distribution of meteorological parameters is achieved. The spatial resolution depends on the ray density, grid-spacing and travel time precision. In Fig. 3 a theoretical boundary for the minimum size of resolvable anomaly according to a formula of Krajewski et al. (1989) is illustrated. When the measured travel times are accurate and the temperature inhomogeneities are large, small anomalies can be resolved. The grid cell size was chosen according to a criterion suggested by Krajewski et al. (1989); specifically the cell size is about equal to the minimum dimension of the resolvable anomalies.

Because of the actual measuring arrangement, not all grid cells are traversed by a sufficiently high number of sound rays. The poorly sampled regions are filled with additional values from fictitious sources and receivers,

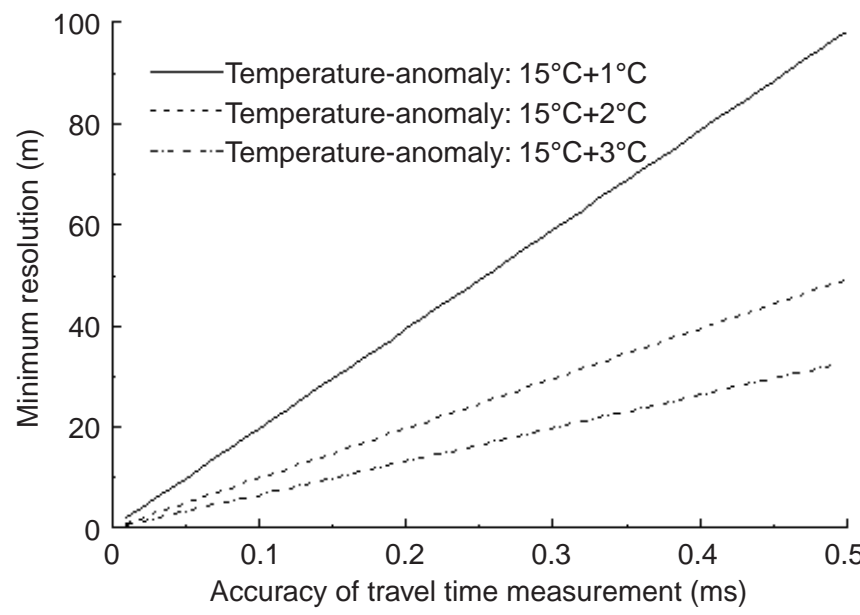

Fig. 3. Theoretical bounds for the minimum size of the resolvable anomaly according to Krajewski et al. (1989), depending on the accuracy of travel time measurements for different values of a temperature anomaly, and an undisturbed value of air temperature of $15{ }^{\circ} \mathrm{C}$ created using geometrical relations. Two travel time measurements are the starting points. Additional values are calculated for fictitious sources and receivers between the real ones using the averaged travel time data for fictitious ray paths.

\section{Experimental procedure and data analyses}

The experimental studies should answer the following questions:

1. What accuracy is attainable to estimate meteorological parameters and what additional information is necessary?

2. Is there a possibility of producing absolute data (temperature and wind field) directly without additional information, or only fluctuations to a known initial state, (see Spiesberger and Fristrup 1990 and Wilson and Thomson 1994)?

To obtain meteorologically relevant data (temperature $\pm 0.3 \mathrm{~K}$, wind velocity $\pm 0.5 \mathrm{~ms}^{-1}$ ), many requirements are obligatory. The demanded accuracy for the travel time measurements can be derived in a manner similar to Spiesberger and Fristrup (1990). The travel time difference between the actual travel time $\tau_{1}$ along ray path $\Gamma_{1}$, and the undisturbed (without wind influence) reference travel time $\tau_{0}$ along the ray path $\Gamma_{0}$ is given by:

$\tau_{1}-\tau_{0}=\int_{\Gamma_{1}} \frac{\mathrm{d} l}{c_{1}\left(\Gamma_{1}\right)+\mathbf{u}\left(\Gamma_{1}\right) \cdot \mathbf{l}}-\int_{\Gamma_{0}} \frac{\mathrm{d} l}{c_{0}\left(\Gamma_{0}\right)}$,

where $\mathbf{u}$ is the wind vector, $\mathbf{I}$ is the unit vector along the ray path, $\mathrm{d} l$ is the differential length of the path and $c_{0}$ and $c_{1}$ are the sound velocities. Here it will be assumed that $c_{1}=c_{0}+\delta c$. Assuming approximately equal ray paths leads to:

$\tau_{1}-\tau_{0}=-\int_{\Gamma_{0}} \frac{\mathrm{d} l(\delta c+\mathbf{u} \cdot \mathbf{l})}{c_{0}^{2}+c_{0} \delta c+c_{0}(\mathbf{u} \cdot \mathbf{l})}$.

Figure 4 illustrates the great influence of the sourcereceiver distance for the demanded travel time accuracy computed with Eq. (12) corresponding to temperature influence without wind on one side and with wind influence without temperature changes on the other side. The travel time measurements have to be carried out with a high degree of accuracy. This requirement includes, as pointed out already, precise determination of the distance between source and receiver, synchronisation of all connected instrumentation, and data processing. Additionally, separation of different effects on travel time is necessary. A single travel time measurement contains mixed information on temperature and the component of wind along the path. With the rough approximation of a reciprocal sound transmission along the same path a separation of the two effects for a first view is possible.

To test the experimental design a field experiment was made in autumn 1996 (Raabe et al., 1996). The 


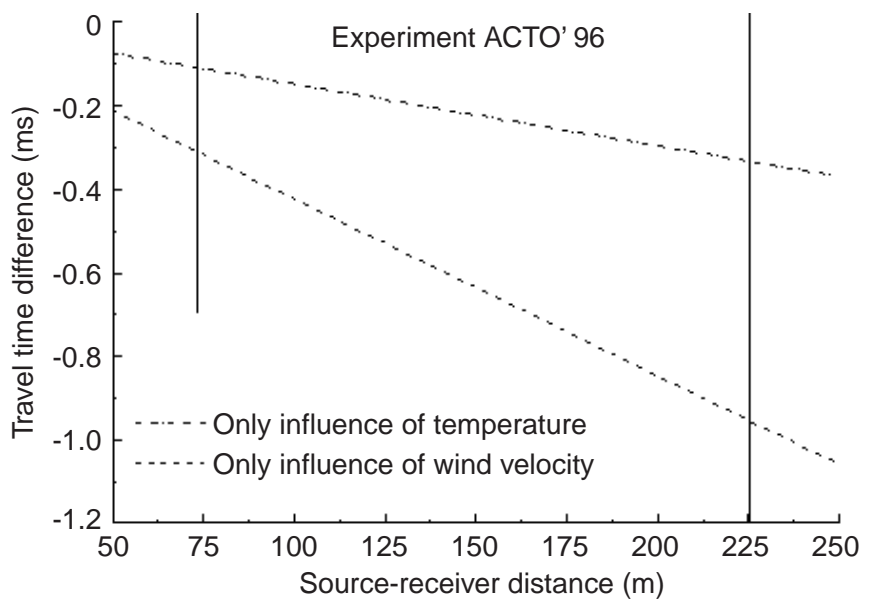

Fig. 4. Required travel time accuracy corresponding to the influence of temperature alone (air temperature: $15^{\circ} \mathrm{C}$, desired accuracy: $0.3 \mathrm{~K}$ ) and to the wind influence alone (wind velocity: $0.5 \mathrm{~ms}^{-1}$, desired accuracy: $0.5 \mathrm{~ms}^{-1}$ ) for source-receiver distances of the experimental campaign ACTO'96

measurements were carried out on a grass-covered plain inside a racecourse in the city of Leipzig. Figure 1 shows the layout of the tomographic array of $100 \times 200 \mathrm{~m}^{2}$. Four sources (compression drivers) and three receivers were positioned on tripods at a height of $2 \mathrm{~m}$. The positions of the transducers were determined using trigonometric measurements with an accuracy of $0.1 \mathrm{~cm}$. The effects of the extension of the technical device (speaker: $\pm 5 \mathrm{~cm}$, microphone $\pm 1 \mathrm{~cm}$ ) can be neglected during the post processing calculations. Over various short distances (between $3 \mathrm{~m}$ and $10 \mathrm{~m}$ ) the transmitted signal was recorded and from the shift of a marked point the influence of the speaker extension can be separated.

All sources simultaneously transmit an acoustic signal. The signal is a sine oscillation with a constant frequency of $500 \mathrm{~Hz}$. The duration of the signal was chosen to be very short (4 ms) to prevent overlapping of signals. However the ringing of the compression driver prolongs the signal. The function generator is additionally connected with all data loggers and gives the start signal for registration. So synchronisation of all devices is ensured.

A receiver set consists of an one inch microphone, a sound level meter and a data logger. The sound level meter is used for power supply and band-pass filtering. After amplifying the signals were sent to the A/D converter. The 10 bit converter was capable of sampling at a rate of $10 \mathrm{kHz}$. The digitised data were stored on a memory card and after each experimental session transferred to a hard drive (laptop).

The travel time of each signal was estimated from the recorded data by cross correlation between the received (output) and the transmitted (input) signal. Each peak of the cross correlation is associated with a separate ray path. The delay time corresponds to the travel time of the transmitted signal. Using the input signal as a calibration signal, all delays caused by the device can be eliminated. Figures 5 and 6 show an example of the recorded signals and the cross correlation of the filtered signal with the reference signal. Figure 5 indicates good signal-to-noise ratio for all distances.

Except for the ray paths S2-R2 and S3-R2, all single rays have significantly different path lengths. As a result, the assignment for each signal to the corresponding source is possible on account of the tomographic array layout. The effect of ground reflection can be roughly estimated from the geometrical travel time difference between the direct and the reflected sound ray according to Klug (1991). Thereby, a constant sound speed $c$ in the atmosphere is assumed:

$\Delta t_{\text {geom }}=\frac{\sqrt{\left(h_{S}+h_{R}\right)^{2}+D^{2}}}{c}-\frac{\sqrt{\left(h_{S}-h_{R}\right)^{2}+D^{2}}}{c}$

In Fig. 7 the travel time difference between the ground reflected and the direct sound ray is displayed depending on the source-receiver distance $D$ with a source height $h_{S}$ of $2 \mathrm{~m}$, a receiver height $h_{R}$ of $4 \mathrm{~m}$ and a height-constant

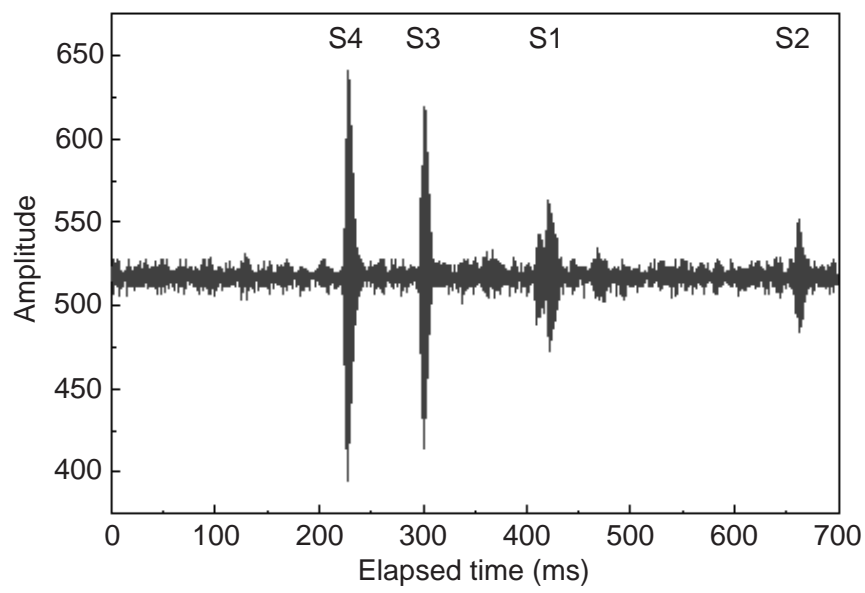

Fig. 5. Example of recorded signals (receiver R1) after bandpass filtering during the tomography experiment on the 7 October, 1996, 18.37 MET

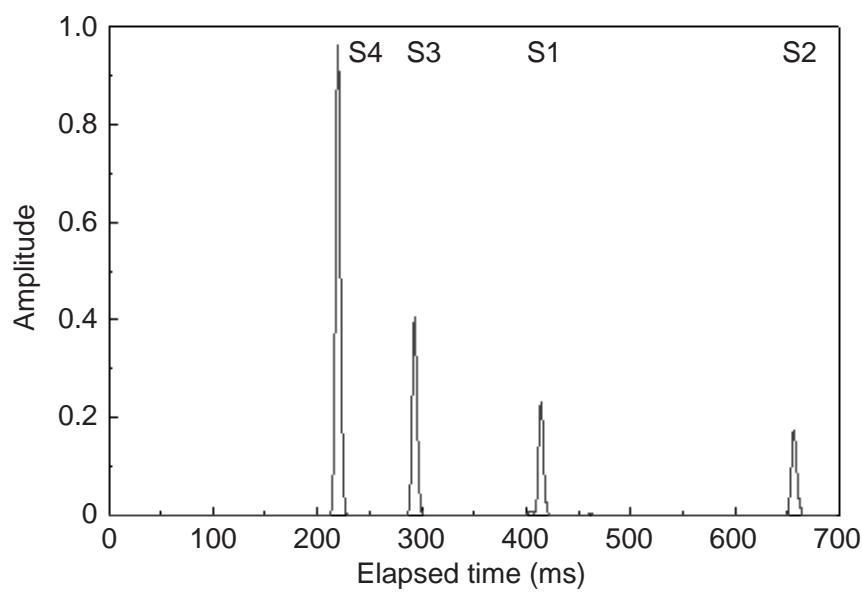

Fig. 6. Cross correlation of the filtered signal (Fig. 5) with the reference signal 


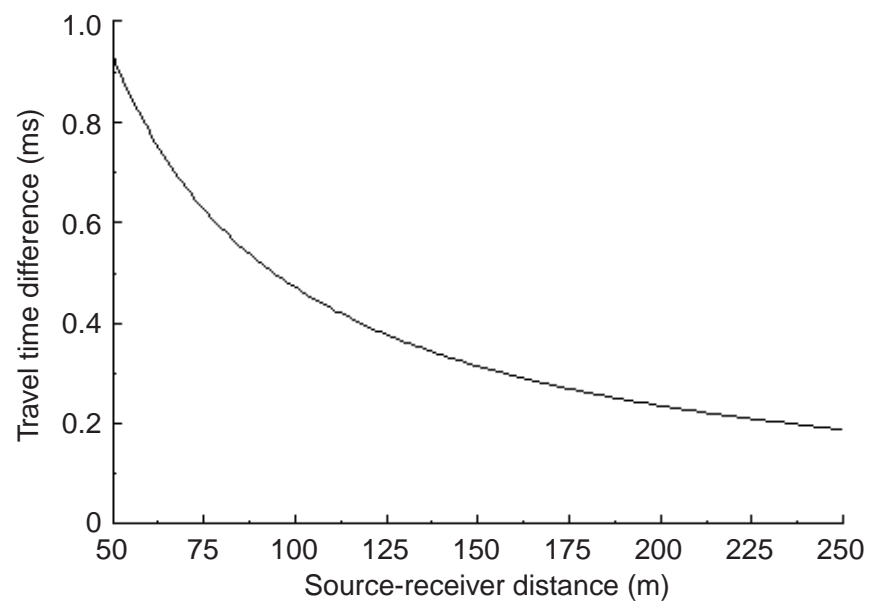

Fig. 7. Travel time difference between the ground reflected and the direct sound ray according to Klug (1991), as a function of the sourcereceiver distance. The source height is $2 \mathrm{~m}$, the receiver height $4 \mathrm{~m}$, and the height-constant sound speed is $339 \mathrm{~ms}^{-1}$

sound speed of $339 \mathrm{~ms}^{-1}$. The larger the source-receiver distance is, the smaller the path and travel time difference will be. In this case the travel times can only be distinguished using information about the sound amplitude, which is smaller for the ground reflected ray over grassland than for the direct sound ray.

Additional to the acoustic experiments meteorological measurements were carried out (see Schienbein and Arnold, 1996). To simulate the actual sound propagation in the atmospheric surface layer with the described ray-tracing model wind and temperature profiles were simultaneously measured. On the meteorological mast (12 $\mathrm{m}$ high) 6 cup anemometers and 6 temperature sensors were fixed at different levels above the ground.
Furthermore, measurements of wind and temperature accomplished with an ultrasonic anemometer were used for the validation of the tomographic model and to compare the area-averaged data with point measurements.

\section{Results}

Area-averages of the effective sound velocity, with one value for each grid cell, were calculated with travel time data from the introduced experimental campaign (see Fig. 8). Although the obtained values have not been recalculated yet into meteorological parameters, general temperature trends are visible. The cooling phase on the 3 , October is reproduced through decreased values of the sound velocity.

For validation of the measurements the travel times between source and receiver were inverted into an average temperature over its propagation path neglecting the wind influence. Comparison between the individual acoustically estimated temperatures and the over all single propagation paths averaged temperatures demonstrates the quality of the measurements. Figure 9 illustrates good correspondence between the individual data and the averaged temperature. However, the deviation of the individual measurements is unsatisfactory. Except the influence of the turbulence, this was mainly caused by one basic restriction of these measurements. The mixed information on sound speed depending on the actual temperature and the wind component along the path can not be satisfactory separated at present.

A comparison between the point measurements, accomplished with a ultrasonic anemometer and the area-averaged calculations was made for the tempera-

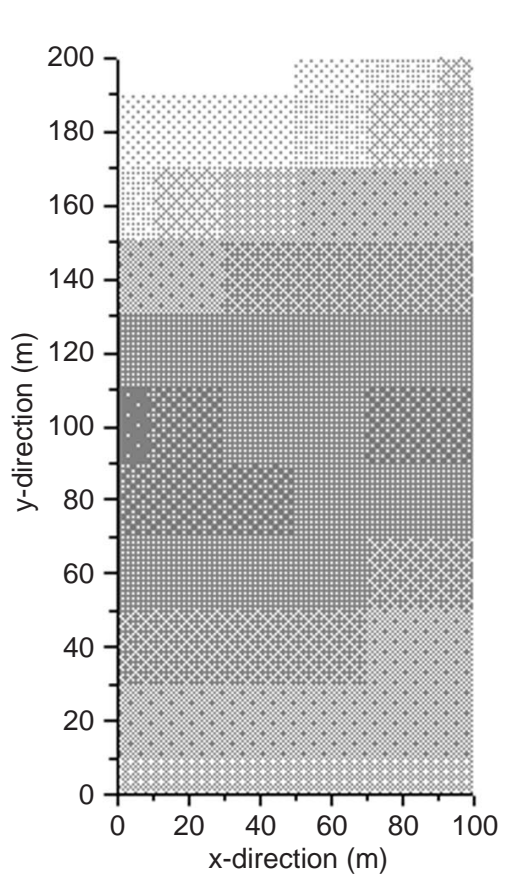

Sound speed $\left(\mathrm{ms}^{-1}\right)$

$340.4-340.6$

$340.2-340.4$

$340.0-340.2$

$339.8-340.0$

$339.6-339.8$

$339.4-339.6$

$339.2-339.4$

$339.0-339.2$

$338.8-339.0$

$338.6-338.8$

$338.4-338.6$

$338.2-338.4$

$338.0-338.2$

$337.8-338.0$

$337.6-337.8$

$337.4-337.6$

$337.2-337.4$

$337.0-337.2$

$336.8-337.0$

$336.6-336.8$

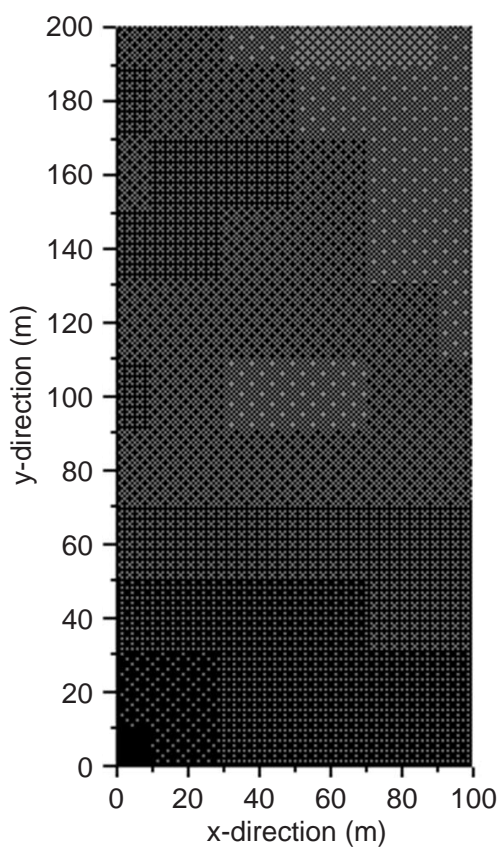

Fig. 8. Area averaged values (one constant for each grid cell) for the effective sound speed from acoustic travel time tomography. Left: 03.10.1996, 17.15 MET; right: 03.10.1996, 18.45 MET 


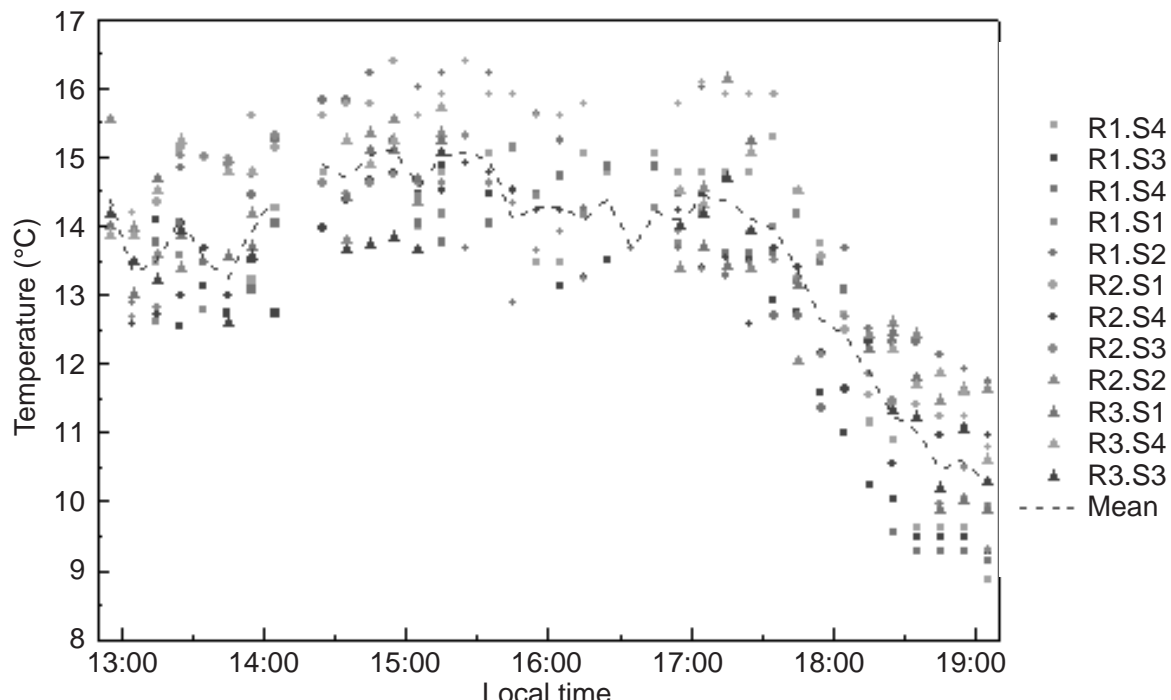

Fig. 9. Comparison between temperature measurements calculated from all possible ray paths and from an average overall single propagation paths on the 3 October, 1996 ture on the 3 October, 1996. The temporal development of distinctive radiant cooling in the evening corresponds well with the acoustic data (see Fig. 10). In testing the procedure the accuracy of the acoustic monitoring was estimated depending on the range between source and receiver. For distances up to $200 \mathrm{~m}$ the precision of the measurements amount to $\pm 0.5 \mathrm{~K}$ for the temperature and $\pm 1 \mathrm{~ms}^{-1}$ for the wind velocity. With increasing distances (the equipment used allows distances up to $500 \mathrm{~m}$ ) the degree of accuracy also increases.

\section{Conclusions and outlook}

Our initial evaluation, although modest in scale, demonstrates the general applicability of tomographic monitoring. Acoustic measurements alone are insufficient for the tomographic studies presented. Additional information about wind and temperature profiles are necessary to simulate the general sound propagation in

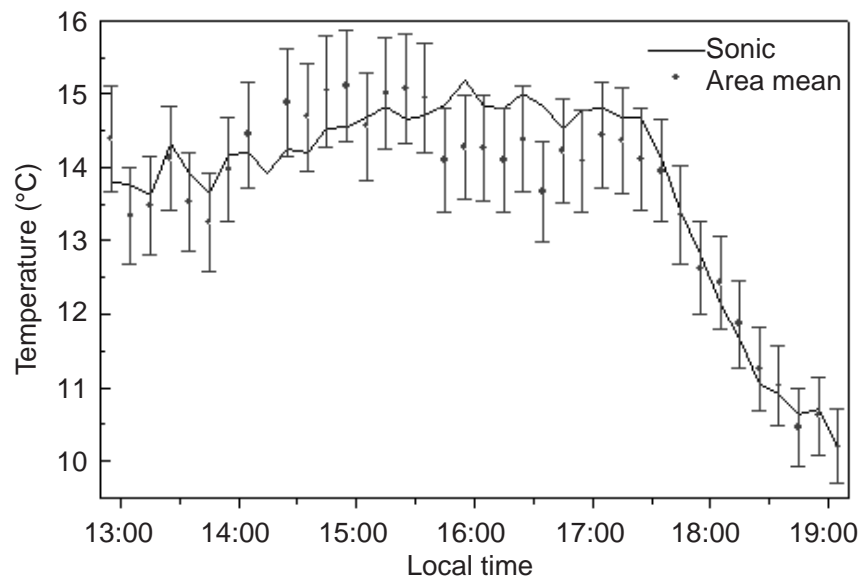

Fig. 10. Comparison between the point measurements, accomplished with a ultrasonic anemometer, and the area-averaged calculations for the air temperature on the 3 October, 1996. The error bars indicate the accuracy of the acoustic monitoring the atmospheric surface layer under different meteorological conditions and to validate the tomographic data.

For meteorologically relevant data a higher precision of the procedure is necessary. At present area-averages for sound speed could be converted with sufficient accuracy for special applications into meteorological parameters, i.e., $0.5 \mathrm{~K}$ for temperature and $1 \mathrm{~ms}^{-1}$ for wind velocity. However, the following improvements are indispensable: further perfecting of the measuring configuration (e.g. an increased height of the experimental equipment to decrease the influence of the ground); improved calculation of the wind influence on the travel time measurements; and also increased number of sources and receivers to achieve better area coverage. Furthermore, increasing the sampling rate results in a higher degree of accuracy for the travel time estimation. To date only a straightforward sound propagation and area-averaged values can be considered with a twodimensional $(x-y)$ tomographic model. Therefore improvements with regard to an expansion in the vertical model direction have to be applied in order to get volume averaged values for meteorological parameters.

Acknowledgements. We would like to thank F. Weiße and M. Engelhorn for their support in the development and manufacturing of the measuring system. Furthermore, we especially acknowledge the help of S. Schienbein for maintenance of the meteorological mast during the experimental campaign. Special thanks also go to E. Danckwardt from the Institute of Geophysics at the University of Leipzig, for support during the application of the tomographic model to the atmosphere. We also thank the students of the Institute of Meteorology for their assistance during the experiment. Last but not least we wish to thank D.K. Wilson from the US Army Research Laboratory and the anonymous reviewer for constructive remarks to the manuscript. This work was supported by the Deutsche Forschungsgemeinschaft under grant Ra 569/3-1.

Topical Editor J. -P. Duvel thanks D. K. Wilson and another referee for their help in evaluating this paper.

\section{References}

Aki, K., and P. Richards, Quantitative seismology-Theory and methods, vol II, W. H. Freeman., 1980. 
Aubry, M., F. Baudin, A. Weill, and P. Rainteau, Measurements of the total attenuation of acoustic waves in the turbulent atmosphere, J. Geophys. Res., 79, 5598-5606, 1974.

Backus, G., and F. Gilbert, The resolving power of gross earth data, Geophys. J., 16, 169-205, 1968.

Bass H. E., Absorption of sound in air: high temperature predictions, J. Acoust. Soc. Am., 69, 124-138, 1981.

Birkhoff, G., The consistency of models of sound waves in fluids, in Eds. Lee, D., R. L. Sternberg and M. H. Schultz, Computational Acoustics, Wave propagation, Elsevier Science Publishers, 117$156,1988$.

Bording, R. P., A. Gersztenkorn, L. R. Lines, J. A. Scales, and S. Treitel, Applications of seismic travel-time tomography, Geophys. J. R. Astron Soc., 90, 285-303, 1987.

Bregman, N. D., R. C. Bailey, and C. H. Chapman, Crosshole seismic tomography, Geophysics, 54, 200-215, 1989a.

Bregman, N. D., C. H. Chapman, and R. C. Bailey, Travel time and amplitude analysis in seismic tomography, J. Geophys. Res., 94, 7577-7587, 1989b.

Carrion, P., Dual tomography for imaging complex structures, Geophysics, 56, 1395-1404, 1991.

Chapman, C. H., The Radon transform and seismic tomography, in Ed. Nolet, G., Seismic tomography, D. Reidel, Dordrecht, 2547, 1987.

Daigle, G. A., J. E. Piercy and T. F. W. Embleton, Effects of atmospheric turbulence on the interference of sound waves near a hard boundary, J. Acoust. Soc. Am., 64, 622-630, 1978.

Deardorff, J. W., Three-dimensional numerical study of the height and mean structure of a heated planetary boundary layer, Boundary-Layer Meteorol., 7, 81-106, 1974.

Dines, K. A., and R. J. Lytle, Computerised geophysical tomography, Proc. IEEE, 67, 1065-1078, 1979.

Fasold, W., W. Kraak, und W. Schirmer (Eds.), Taschenbuch der Akustik, Teil 1, VEB Verlag Technik, Berlin, 1984 (in German).

Gelbke, C., Seismische Durschallungstomographie, Technischer Bericht Felslabor Grimsel NAGRA NTB 88-06, 3-42, 1988 (in German)

Gilbert, P., Iterative methods for the three-dimensional reconstruction of an object from projections, J. Theor. Biol., 36, 105117, 1972.

Gordon, R., R. Bender, and G. T. Herman, Algebraic reconstruction techniques for three-dimensional electron microscopy and Xray photography, J. Theor. Biol., 29, 471-481, 1970.

Herman, G. T., Reconstructions from projections - The fundamentals of computerized tomography, Academic Publishing, San Diego, 1980.

Humphreys, E., and R. W. Clayton, Adaptation of back projection tomography to seismic travel time problems, J. Geophys. Res., 93, 1073-1085, 1988.

Ivansson, S., Remark on earlier proposed iterative tomographic algorithm, Geophys. J., 75, 855-860, 1983.

Kaczmarz, S., Angenäherte Auflösung von Systemen linearer Gleichungen, Bull. Acad. Polon. Sci. Lett., A 35, 355-357, 1937 (in German).

Klug, H., Sound-speed profiles determined from outdoor sound propagation measurements, J. Acoust. Soc. Am., 90, 475-481, 1991.

Kneser, H. O., Schallabsorption und -dispersion in Gasen, 192 195, in Handbuch der Physik, Band XI/1, Springer-Verlag, Berlin, 1961 (in German).

Kornhauser, E. T., Ray theory for moving fluids, J. Acoust. Soc. Am., 25, 945-949, 1953.

Krajewski, C., L. Dresen, C. Gelbke, and H. Rüter, Iterative tomographic methods to locate seismic low-velocity anomalies: a model study, Geophys. Prosp., 37, 717-751, 1989.
Lo, Tien-when and P. L. Inderwiesen, Fundamentals of seismic tomography, Geophysical Monograph Series, 6, Society of Exploration Geophysicists, 1994.

McMechan, G. A., Seismic tomography in boreholes, Geophys. J. R. Astron. Soc., 74, 601-612, 1983.

Mix, W., V. Goldberg, and K. -H. Bernhardt, Numerical experiments with different approaches for boundary layer modeling under large-area forest canopy conditions, Meteorol. Z., N.F. 3, 187-192, 1994.

Moeng, C. -H., A large-eddy-simulation model for the study of planetary boundary-layer turbulence, J. Atmos. Sci., 41, 20522062, 1984.

Munk, W. H., P. Worcester, and C. Wunsch, Ocean acoustic tomography, Cambridge University Press, New York, 1995.

Peterson, J. E., B. N. P. Paulsson, and T. V. McEvilly, Applications of algebraic reconstruction techniques to crosshole seismic data, Geophysics, 50, 1566-1580, 1985.

Pierce, A. D., Acoustics. An introduction to its physical principles and applications, Acoustical Society of America, New York, 1989.

Raabe, A., K. Arnold, and A. Ziemann, Akustische Tomographie im Bereich der Atmosphärischen Grenzschicht, Wiss. Mitt. Inst. für Meteorol. Univ. Leipzig und Inst. für Troposphärenforschg. Leipzig, 4, 113-123, 1996 (in German).

Radon, J., Über die Bestimmung von Funktionen durch die Integralwerte längs gewisser Mannigfaltigkeiten, Berichte Sächs. Akademie der Wiss., 69, 262-277, 1917 (in German).

Rüter, H., and C. Gelbke, Seismische Tomographie, in Eds. Dresen, L., J. Fertig, H. Rüter and W. Budach: 6. Minitrop-Seminar. Seismik auf neuen Wegen. Ausgewählte Beispiele und Schwerpunkte, DVGI-Fachauschuß Geophysik Celle, 207-240, 1986 (in German).

Santamarina, J. C., and A. C. Reed, Ray tomography: errors and error functions, J. Appl. Geophys., 32, 347-355, 1994.

Schienbein, S., and K. Arnold, Konzeption und Aufbau einer Meßeinrichtung für die Erfassung von Temperaturen und Strömungsgeschwindigkeiten bis $12 \mathrm{~m}$ Höhe, Wiss. Mitt. Inst. für Meteorol. Univ. Leipzig und Instit. für Troposphärenforschg. Leipzig, 4, 124-130, 1996 (in German).

Shaw, R. H., and U. Schumann, Large-eddy simulation of turbulent flow above and within a forest, Boundary-Layer Meteorol., 61, 47-64, 1992.

van der Sluis, A., and H. A. van der Vorst, Numerical solution of large, sparse linear algebraic systems arising from tomographic problems, in Ed. Nolet, G., Seismic tomography, D. Reidel, Dordrecht, 70, 49-83, 1987.

Spiesberger, J. L., and K. M. Fristrup, Passive localization of calling animals and sensing of their acoustic environment using acoustic tomography, Am. Natural., 135, 107-153, 1990.

Tatarskii, V. I., Wave propagation in a turbulent medium, McGrawHill, New York, 1961.

Trampert, J., and J. -J. Leveque, Simultaneous iterative reconstruction technique: Physical interpretation based on the generalized least squares solution, J. Geophys. Res., 95, 1255312559,1990

Ugincius, P., Acoustic-ray equations for a moving, inhomogeneous medium. J. Acoust. Soc. Am., 37, 476-479, 1965.

Wilson, D. K., and D. W. Thomson, Acoustic tomographic monitoring of the atmospheric surface layer, J. Atmos. Ocean. Technol., 11, 751-768, 1994.

Worthington, M. H., An introduction to geophysical tomography, First Break, 2, 20-26, 1984.

Ziemann, A., Numerical simulation of meteorological quantities in and above forest canopies, Meteorol. Z., N.F. 7, 120-128, 1998. 